\title{
MicroRNA-21 modulates radiation resistance through upregulation of hypoxia-inducible factor-1 $\alpha$-promoted glycolysis in non-small cell lung cancer cells
}

\author{
SHUMEI JIANG* , RENBEN WANG* , HONGJIANG YAN, LINZHI JIN, XUE DOU and DONG CHEN
}

Department of Radiation Oncology, Shandong Cancer Hospital and Institute, Jinan, Shandong 250117, P.R. China

Received December 29, 2014; Accepted September 25, 2015

DOI: $10.3892 / \mathrm{mmr} .2016 .5010$

\begin{abstract}
Aberrant microRNA (miRNA) expression in cancer affects the transcription of target genes, and profoundly influences cancer-associated signaling pathways. Radiation resistance is a major problem encountered in the treatment of cancer. The present study aimed to investigate the role of miRNA (miR)-21 in the development of radiation resistance in non-small cell lung cancer cells. A radiation-resistant cell line was generated from A549 cells. Significant upregulation of miR-21 was detected in the radioresistant cancer cells, as compared with the radiosensitive cells, and overexpression of miR-21 rendered A549 parental cells resistant to radiation. In addition, glycolysis was increased in the radioresistant cells, as compared with the sensitive cells. Furthermore, hypoxia-inducible factor- $1 \alpha$ (HIF $1 \alpha$ ) was upregulated by miR-21 in radioresistant cells, resulting in promotion of the key enzymes of glycolysis. Inhibition of HIF1 $\alpha$ by small interfering RNA suppressed glycolysis and resensitized the cancer cells to radiation, whereas the recovery of HIF1 $\alpha$ in miR-21-inhibited radioresistant cells resulted in recovery of radioresistance. In conclusion, the present study suggested that miR-21 may modulate radioresistance through the upregulation of HIF1 $\alpha$. These results may provide a novel perspective on miRNA for the development of anti-radioresistance drugs.
\end{abstract}

\section{Introduction}

MicroRNAs (miRNAs) are functional non-coding RNAs, 20-24 nucleotides long, which have important roles in the regulation of the stability and translational efficiency of target

Correspondence to: Dr Shumei Jiang or Mr. Renben Wang, Department of Radiation Oncology, Shandong Cancer Hospital and Institute, 440 Jiyan Road, Jinan, Shandong 250117, P.R. China

E-mail: shumeijiangsdchi@163.com

E-mail: renbenwangsdchi@163.com

*Contributed equally

Key words: microRNA, micrRNA-21, radioresistance, non small cell lung cancer, hypoxia-inducible factor- $1 \alpha$, glycolysis
mRNAs (1). Mature miRNAs induce post-transcriptional gene silencing by targeting the complementary sequence motifs within the 3'-untranslated regions of target mRNAs. Therefore, aberrant miRNA expression may affect the transcription of target genes and profoundly influence cancer-associated signaling pathways, which are involved in proliferation, cell cycle control, apoptosis, differentiation, migration and metabolism (2,3). Tumor tissue usually exhibits reduced levels of mature miRNAs (4); however, miRNA (miR)-21 has been reported to act as an oncomiR, which is highly expressed and correlated with numerous types of cancer, including pancreatic (5), breast (6), non-small cell lung (7), liver (8), gastric (9), ovarian (10), cervical (11), colon (12), brain (13), esophageal (14) and prostate (15) cancer. In addition, a previous study demonstrated that miR-21 modulated paclitaxel sensitivity in ovarian cancer (16), indicating that miR-21 has an important role in chemoresistance.

Ionizing radiation is used extensively to treat various types of cancer. However, despite the wide use of radiation therapy and its association with remission, tumor recurrence is a predominant cause of radiation treatment failure, and is frequently associated with the acquisition of radioresistance by tumors (17). At present, the detailed mechanism underlying radiation resistance in human cancer remains unclear. It has previously been reported that upregulated phopshoinositide 3-kinase/AKT signaling may render human liver and cervical cancer resistant to radiation $(17,18)$. In addition, a previous study demonstrated that high expression levels of miR-21 may contribute to radiation resistance in breast cancer cells (19), thus suggesting that targeting miR-21 may be considered a therapeutic strategy to overcome radiation resistance.

The majority of cancer cells prefer anaerobic glycolysis for the production of their energy supply, which is known as the 'Warburg effect'; however, anaerobic glycolysis is less efficient at producing ATP, as compared with mitochondrial respiration (20). As a result, uptake of excessive glucose and generation of large quantities of lactate by cancer cells is a well-recognized phenotype of abnormal glycolysis. Notably, previous studies have suggested that metabolic pathways are associated with chemoresistance $(17,18,21)$, suggesting that dysregulated metabolic pathways in cancer may be a target for the development of novel therapeutic strategies. The present study identified the role of $\mathrm{miR}-21$ in radiation resistance. The results detected a correlation between glycolysis and 
radioresistance, and may provide evidence regarding the mechanisms underlying miR-21-mediated radioresistance in non-small cell lung cancer cells.

\section{Materials and methods}

Cell culture and ionizing radiation treatment. The A549 lung cancer cells were obtained from the American Type Culture Collection (Manassas, VA, USA) and cultured in RPMI-1640 media (Gibco; Thermo Fisher Scientific, Inc., Waltham, MA, USA) supplemented with $10 \%$ fetal bovine serum (Thermo Fisher Scientific, Inc.) and antibiotics (Gibco Antibiotic-Antimycotic containing mphotericin B, penicillin and streptomycin; Gibco, Thermo Fisher Scientific, Inc.) at $37^{\circ} \mathrm{C}$ in a humidified incubator containing $5 \% \mathrm{CO}_{2}$. The cells, at a density of $5 \times 10^{5}$, were exposed to various doses of irradiation $(0,0.5,1,2,4,5,6,8$ and $10 \mathrm{~Gy})$ using a Cs-137 irradiator (HWM D-2000; Siemens, Waltershausen, Germany) at a dose rate of $2 \mathrm{~Gy} / \mathrm{min}$. Irradiation was administered at room temperature and the cells were subsequently incubated at $37^{\circ} \mathrm{C}$, and harvested for the following experiments.

Antibodies. The following antibodies were used in the present study: Anti-hexokinase 2 (HKII) (cat. no. 2867), anti-pyruvate kinase (PK)M2 (cat. no. 4053), anti-lactate dehydrogenase (LDH)A (cat. no. 2012), anti- $\beta$-actin (cat. no. 4967) (Cell Signaling Technology, Inc., Danvers, MA, USA), and anti-hypoxia-inducible factor (HIF) $1 \alpha$ (cat. no. sc-10790; Santa Cruz Biotechnology, Inc., Dallas, TX, USA).

Small interfering (si)RNA and plasmid DNA transfection. HIF-1 $\alpha$ siRNA sequences (cat. no. 106498) and negative control siRNA were purchased from Ambion (Thermo Fisher Scientific, Inc.). Cells were seeded onto 6-well plates at a density of $1 \times 10^{5}$ cells/well. A plasmid vector containing wild type HIF1 $\alpha$ was purchased from Addgene (Cambridge, MA, USA). Transfection was performed using Lipofectamine ${ }^{\circledR} 2000$ and Opti-MEM I reduced serum medium (Invitrogen; Thermo Fisher Scientific, Inc.), according to the manufacturer's protocol. Transfection temperature was $37^{\circ} \mathrm{C}$, and the volume of siRNA/vector and Lipofectamine used was $100 \mathrm{nM}$ siRNA/vector and $5 \mu \mathrm{l}$ Lipofectamine, respectively for each transfection. A total of 48 hours post-transfection, the cells were prepared for further analysis.

miRNA transfection. The precursor and antisense sequences of miR-21 were chemically synthesized by GenePharma Co., Ltd. (Shanghai, China). The cells were seeded in 6-well plates at $1 \times 10^{5}$ cells/well and cultured overnight. Subsequently, the cells were transfected with $200 \mathrm{nM}$ pre-miR-21, inhibitors or negative control, using Lipofectamine ${ }^{\circledR} 2000$ and Opti-MEM I reduced serum medium (Invitrogen), according to the manufacturer's protocol. A total of 48 hours post transfection, the cells were prepared for further analysis.

Cell viability assay. The cells were seeded into 96-well culture plates at a density of $5 \times 10^{3}$. Following cellular adhesion, the cells were exposed to various doses of irradiation. Following irradiation, $20 \mu 15 \mathrm{mg} / \mathrm{ml} \mathrm{3-(4,5-dimethylthiazol-2-yl)-2,5-di-}$ phenyltetrazolium bromide (MTT; Sigma-Aldrich, St. Louis,
MO, USA) was added to each well. Following a $4 \mathrm{~h}$ incubation at $37^{\circ} \mathrm{C}$, the medium was gently aspirated and replaced with $150 \mu$ l dimethylsulfoxide. The absorbance of each well was detected at a wavelength of $570 \mathrm{~nm}$ using an ELx800 Absorbance Reader (BioTek Instruments, Inc., Winooski, VT, USA). The experiment was conducted in triplicate.

RNA extraction and reverse transcription-quantitative polymerase chain reaction ( $R T-q P C R)$. Total RNA was extracted from the cells using the Absolutely RNA RT-PCR Miniprep kit (Agilent Technologies, Inc., Santa Clara, CA, USA), according to the manufacturer's protocol. Total RNA concentration was adjusted to $2 \mathrm{ng} / \mu \mathrm{l}$ using a NanoDrop 2000 spectrophotometer (Thermo Fisher Scientific, Inc.). Total RNA $(1 \mu \mathrm{g})$ was reverse transcribed using the High Capacity cDNA Reverse Transcription kit (Applied Biosystems; Thermo Fisher Scientific, Inc.). The cDNA was then diluted to 1:10 for use as a template for RT-qPCR. PCR amplifications were performed in a final reaction volume of $10 \mu \mathrm{l}$ containing: $5.5 \mu \mathrm{l}$ TaqMan Universal PCR Master mix (Applied Biosystems), $0.5 \mu 1$ primers and probes mix and $4.5 \mu \mathrm{g}$ cDNA. The cycling conditions were as follows: One cycle at $50^{\circ} \mathrm{C}$ for $2 \mathrm{~min}$, one cycle at $95^{\circ} \mathrm{C}$ for $10 \mathrm{~min}$, followed by 40 cycles of denaturation $\left(95^{\circ} \mathrm{C}\right.$ for $15 \mathrm{sec}$ ), and a final annealing/extension step at $60^{\circ} \mathrm{C}$ for $1 \mathrm{~min}$. All reactions were conducted using the Step One Plus Real-Time PCR Systems Thermocycler (Applied Biosystems). All quantitative PCR reactions were carried out in triplicate and repeated at least twice. The $\Delta \mathrm{C}_{\text {quantification }}\left(\Delta \mathrm{C}_{\mathrm{q}}\right)$ for mRNA expression was calculated relative to the $\mathrm{C}_{\mathrm{q}}$ of $18 \mathrm{~S}$ ribosomal RNA. Relative mRNA expression was calculated using the formula $2^{(-\Delta \Delta \mathrm{Cq})}$. The primers used for qPCR were as follows: LDHA, forward ATCTTGACCTACGTGGCTTGGA and reverse CCATACAGGCACACTGGAATCTC; HKII, forward CAAAGTGACAGTGGGTGTGG and reverse GCCAGGTCC TTCACTGTCTC; and PKM2, forward GAGGCCTCCTTC AAGTGCT and reverse CCAGACTTGGTGAGGACGAT. RT-qPCR quantification using the TaqMan-miRNA assay (Applied Biosystems) was conducted to determine the expression levels of miR-21 (cat. no. 4427975; assay ID 000397), using the cycling conditions previously described. qPCR was performed using the Step One Plus Detection system (Applied Biosystems), according to the manufacturer's protocol. The relative expression values of the specific miRNA were calculated using the $2^{-\triangle \Delta \mathrm{Cq}}$ method, normalized to the control miRNA (GAPDH: 5'-AATCCCATCACCATCTTCCA-3' forward and 5'-TGGACTCCACGACGTACTCA-3' reverse). All reactions were performed at least twice in duplicate.

Measurements of glucose consumption and lactate production. The cells were seeded in 6-well plates at a density of $5 \times 10^{5}$ cells/well in $3 \mathrm{ml}$ culture medium. Then, conditioned medium and distilled water were mixed at a 1:20 ratio (conditioned medium:distilled water. The glucose concentration in the diluted medium was measured using the Glucose Assay kit (Sigma-Aldrich), according to the manufacturer's protocol. Glucose consumption was calculated by subtracting the concentration of glucose remaining in the medium $24 \mathrm{~h}$ later from the concentration of glucose present in the fresh cell culture medium. Lactate concentrations were determined using a Lactate Assay kit (BioVision, Mountain View, CA, 
USA) according to the manufacturer's protocol. Samples and a lactate standard were prepared with lactate assay buffer in a 96-well plate. Subsequently, $50 \mu \mathrm{l}$ lactate enzyme mix was added to each well, and incubated for $30 \mathrm{~min}$ at room temperature. Optical density values were measured at $570 \mathrm{~nm}$ using a microplate reader (SpectraMax M2e; Molecular Devices, LLC, Sunnyvale, CA, USA). The results were normalized to the amount of total protein, as compared with the control cells.

Western blot analysis. The cells were harvested and washed with ice-cold phosphate-buffered saline (PBS). Cell lysates were obtained by re-suspending the cells in radioimmunoprecipitation assay buffer [10 mM Tris (pH 7.4), $150 \mathrm{mM} \mathrm{NaCl}$, $1 \%$ Triton X-100, 1\% Na-deoxycholate (Kanto Chemical Co., Inc., Tokyo, Japan)] and $5 \mathrm{mM}$ EDTA supplemented with protease inhibitor cocktail (Sigma-Aldrich). The protein concentration of the cell lysates was determined by Bradford assay, using a Bradford kit (Beyotime Institute of Biotechnology, Shanghai, China). Equal quantities of protein $(1 \mu \mathrm{g} / \mu \mathrm{l})$ were separated by $10 \%$ homemade sodium dodecyl sulfate-polyacrylamide gel electrophoresis and electrotransferred onto a polyvinylidene difluoride membrane (Bio-Rad Laboratories, Inc., Hercules, CA, USA). The membranes were blocked with $5 \%$ non-fat milk and incubated overnight with primary antibodies at $4^{\circ} \mathrm{C}$ at a dilution of 1:1,000. The membranes were then washed with PBS with Tween 20 and incubated at room temperature for $1 \mathrm{~h}$ with horseradish peroxidase-conjugated goat anti-rabbit immunoglobulin $\mathrm{G}$ secondary antibodies (cat. no., 7074; dilution, 1:3,000; Nichirei Biosciences, Inc., Tokyo, Japan). Protein bands were visualized using Chemi-Lumi One L Western Blotting Substrate (Nacalai Tesque, Kyoto, Japan).

Statistical analysis. Statistical analysis was performed using Prism 5.0 (GraphPad Software, Inc., La Jolla, CA, USA) and the unpaired Student's t-test was used to analyze the data. All data are presented as the mean \pm standard error. $\mathrm{P}<0.05$ was considered to indicate a statistically significant difference.

\section{Results}

miR-21 expression levels are higher in radiation-resistant lung cancer cells. To investigate the association between miR-21 expression and radiation sensitivity, a radiation-resistant non-small cell lung cancer cell line was generated using A549 parental cells, according to a previous study (22). The cells were treated with increasing doses of radiation for 4 months, in order to generate resistant cells. Resistant cell clones were developed and pooled. To confirm that the cells were radioresistant, the A549 parental cells and resistant cells underwent various doses of irradiation. A total of $72 \mathrm{~h}$ post-irradiation cellular proliferation was determined using an MTT assay (Fig. 1A). The A549 radiation-resistant cells were able to tolerate higher intensities of radiation. In addition, the expression levels of miR-21 were significantly upregulated in the radiation-resistant cells, as compared with the sensitive cells (Fig. 1B), thus suggesting that miR-21 may contribute to radioresistance in non-small lung cancer. To confirm the above observation, A549 cells were transiently transfected with pre-miR-21, in order to overexpress miR-21. The radiation sensitivity of A549 cells with and without overexpression of miR-21 were subsequently measured following treatment with the indicated doses. The A549 cells had an increased survival capacity in response to irradiation following transfection with miR-21 (Fig. 1C). These data indicate a strong correlation between miR-21 and radioresistance in lung cancer cells, suggesting that miR-21 may be a target for overcoming radiation resistance.

Radioresistant lung cancer cells exhibit upregulated glycolysis. Enhanced anaerobic glycolysis often accompanies radioresistance in cancer. To determine the mechanisms underlying miR-21-promoted radioresistance, the glycolysis rates of radiation-sensitive and -resistant A549 cells were detected. The glucose consumption and lactate production were determined. As shown in Fig. 2A, glucose consumption and lactate production were promoted in the radiation-resistant cells, thus indicating that upregulated anaerobic glycolysis may be associated with the radioresistance. In addition, the expression levels of glycolysis-associated enzymes were detected. The protein and mRNA expression levels of HKII, PKM2 and LDHA were upregulated in the radioresistant cells (Fig. 2B and C). These results suggest that enhancement of glycolysis by miR-21 is mediated via upregulation of glycolysis-associated enzymes at the transcriptional level.

miR-21 promotes glycolysis via upregulation of HIF $1 \alpha$. To further explore the mechanisms underlying the miR-21-mediated transcriptional regulation of the key enzymes of glycolysis in radiation-resistant cells, a literature search was conducted, focusing on HIF1 $\alpha$, which is a heterodimeric transcription factor induced by hypoxia, growth factors and oncogenes. It has previously been reported that under hypoxic conditions HIF1 $\alpha$ may be activated and stimulate the transcription of downstream target genes, including LDHA and HKII (23). In the present study, it was hypothesized that HIF1 $\alpha$ may be activated in radioresistant cells, and have an important role in radioresistance by promoting the expression of downstream glycolytic enzymes. To determine whether miR-21 affects HIF1 $\alpha$ expression, the A549 radiation-sensitive and -resistant cells were transfected with an miR-21 precursor or control miRNA, and the expression levels of HIF1 $\alpha$ and glycolytic key enzymes were detected, and glucose consumption and lactate production were measured. As hypothesized, overexpression of miR-21 significantly increased the expression levels of HIF $\alpha$ and the key enzymes of glycolysis (Fig. 3A). Consistently, glucose consumption and lactate production were increased following miR-21 transfection (Fig. 3B). To confirm that miR-21 promotes the glycolysis of non-small cell lung cancer cells through the upregulation of HIF $1 \alpha$, HIF $1 \alpha$ expression was silenced in the miR-21-pretransfected cells using siRNA (Fig. 3C). Glucose consumption and lactate production were downregulated following inhibition of HIF1 $\alpha$ (Fig. 3D). These results indicate that miR-21-regulated HIF1 $\alpha$ may be associated with the underlying mechanism of radioresistance.

Suppression of glycolysis by inhibiting miR-21 sensitizes lung cancer cells to radiation. To determine whether miR-21 inhibition was able to enhance the radiosensitivity of lung cancer cells, A549 cells were transfected with antisense oligonucleotides targeting miR-21 or a scrambled 
A

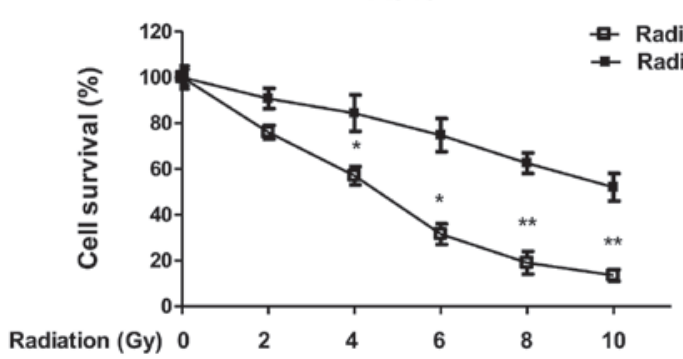

B

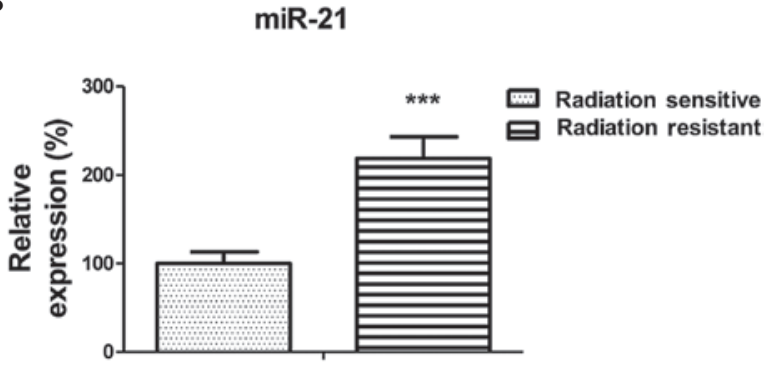

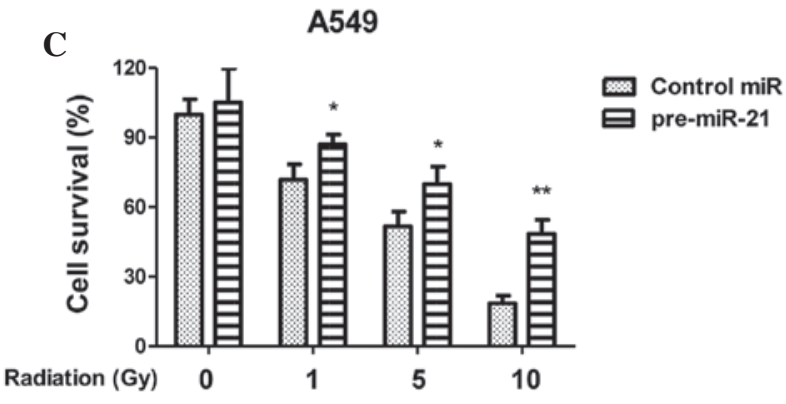

Figure 1. MicroRNA (miR)-21 is upregulated in radioresistant cancer cells and positively correlated with irradiation sensitivity. (A) Generation of radiation-resistant cells from A549 lung cancer cells. Parental cells were treated with gradually increasing doses of irradiation in regular cell culture conditions, in order to generate resistant cells. Radioresistant clones were pooled and analyzed following irradiation at $0,2,4,6,8$ and $10 \mathrm{~Gy}$, at a dose rate of $2 \mathrm{~Gy} / \mathrm{min}$. ${ }^{*} \mathrm{P}<0.05$ and ${ }^{* *} \mathrm{P}<0.01$ versus radiation resistant. (B) Expression levels of miR-21 in A549 parental and radiation-resistant cells. ${ }^{* * *} \mathrm{P}<0.001$ versus radiation sensitive. (C) A549 parental cells were transiently transfected with pre-miR-21 for $48 \mathrm{~h}$, followed by irradiation at $0,1,5$ and $10 \mathrm{~Gy}$, at a dose rate of $2 \mathrm{~Gy} / \mathrm{min}$. A cell survival assay was subsequently performed. ${ }^{*} \mathrm{P}<0.05$ and ${ }^{* * *} \mathrm{P}<0.01$ versus the control. Data are presented as the mean \pm standard error.

A
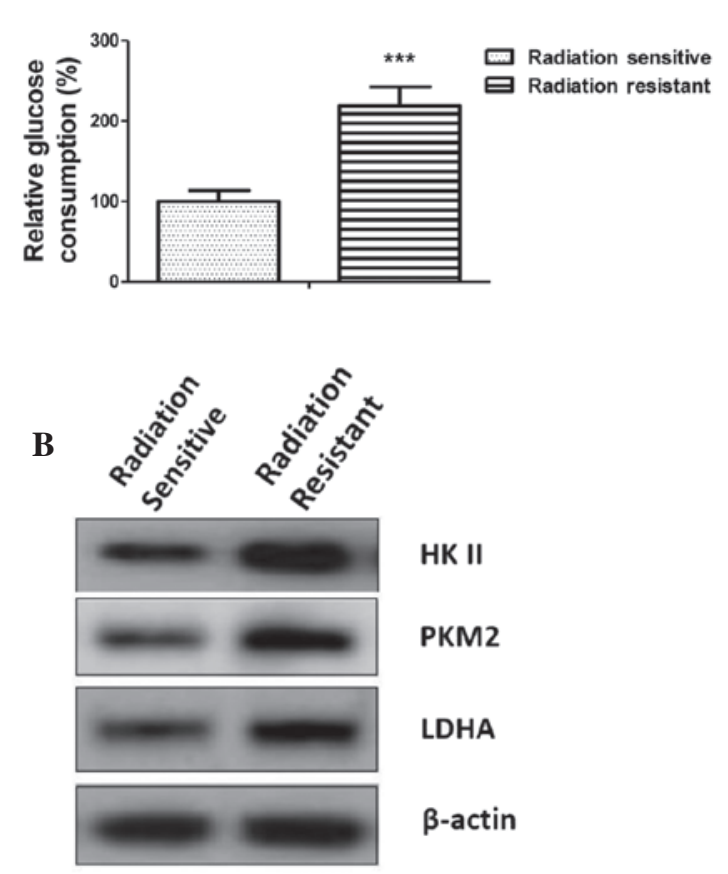

$\beta$-actin

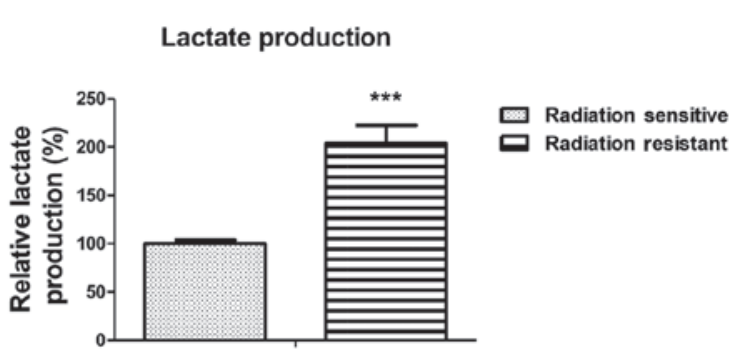

C RT-qPCR

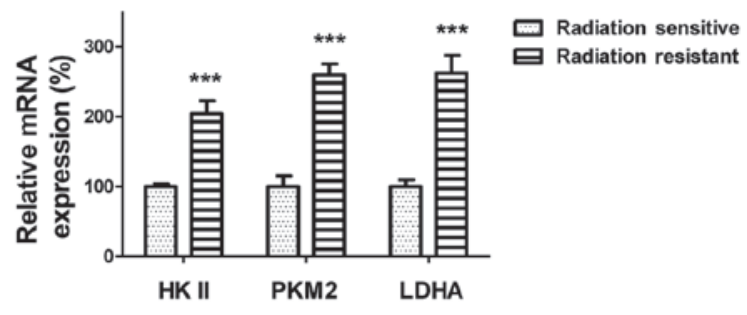

Figure 2. Radiation-resistant cells exhibit upregulated glycolysis. (A) Glucose consumption and lactate production were measured in A549 parental cells and radioresistant cells. (B) Western blotting detected the upregulated protein expression of hexokinase (HK)II, pyruvate kinase (PK)M2 and lactate dehydrogenase (LDH)A in the radiation-resistant cells, as compared with the parental cells. $\beta$-actin was used as a loading control. (C) mRNA expression levels of HKII, PDK1 and LDHA were detected using reverse transcription-quantitative polymerase chain reaction in the A549 parental and radioresistant cells. Data are presented as the mean \pm standard error of three independent experiments. ${ }^{* * *} \mathrm{P}<0.001$ versus radiation sensitive.

miRNA control (Fig. 4A). Glycolysis was measured $48 \mathrm{~h}$ post-transfection. Inhibition of miR-21 decreased glycolysis, as detected by measuring glucose consumption and lactate production (Fig. 4B and C). In addition, A549 cells transfected with anti-miR-21 or control miRNA were exposed to various doses of radiation $(0,0.5,1,2,4$ and $6 \mathrm{~Gy})$ at a rate of $2 \mathrm{~Gy} / \mathrm{min}$, 

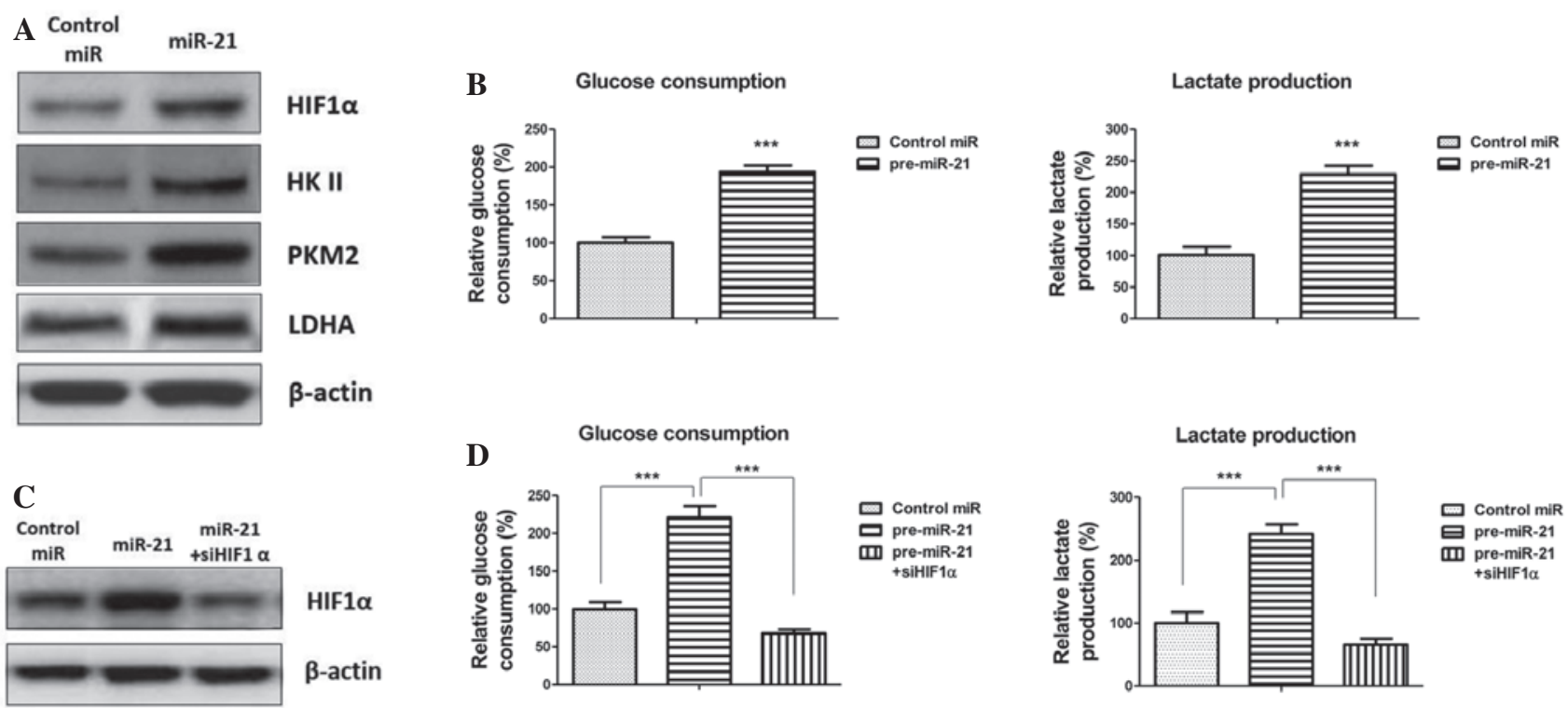

Figure 3. Overexpression of microRNA (miR)-21 upregulates glycolysis through hypoxia-inducible factor (HIF)1 $\alpha$. (A) A549 cells were transiently transfected with pre-miR-21 for $48 \mathrm{~h}$, and the cells were collected and subjected to western blot analysis. $\beta$-actin was used as a loading control. (B) Following pre-miR-21 transfection, glucose consumption and lactate production were measured in the in A549 cells, as compared with the negative control. ${ }^{* * *} \mathrm{P}<0.001$ versus the control. (C) Protein expression levels of HIF1 $\alpha$ were downregulated following small interfering RNA (si)HIF1 $\alpha$ transfection in cells overexpressing miR-21 and control cells, as detected by western blotting. $\beta$-actin was used as a loading control. (D) Glucose consumption and lactate production were measured in cells transfected with control miR, pre-miR-21 or pre-miR-21 plus siHIF1 $\alpha$. Data are presented as the mean \pm standard error. ${ }^{* * *} \mathrm{P}<0.001$. HKII, hexokinase 2 ; PKM2, pyruvate kinase M2; LDHA, lactate dehydrogenase A.

A

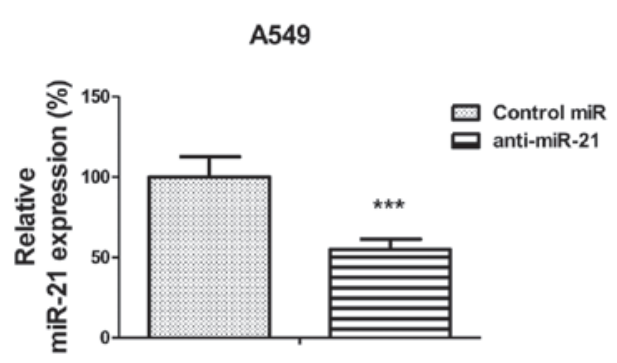

C
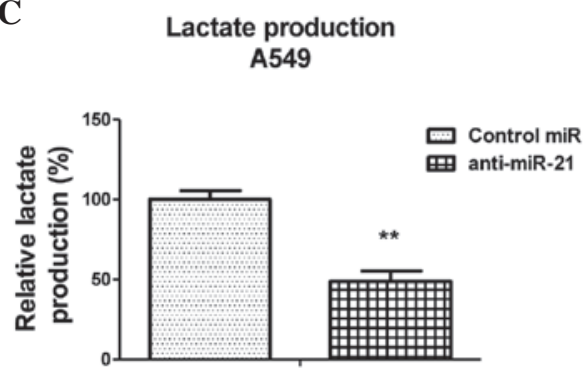

B

Glucose consumption A549

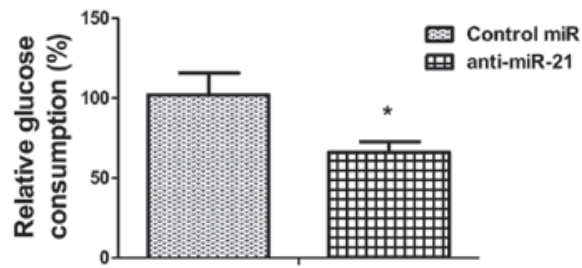

D

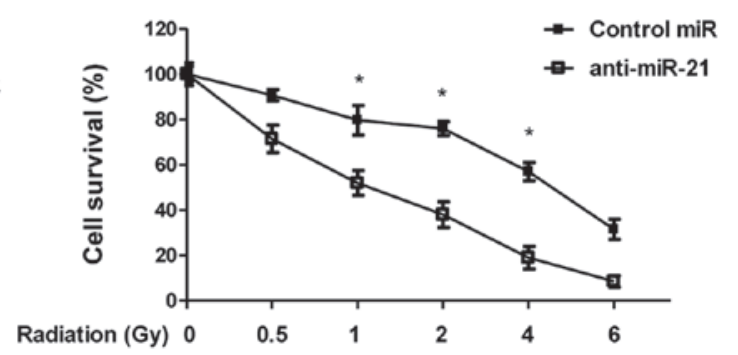

Figure 4. Inhibition of microRNA (miR)-21 suppresses glycolysis and sensitizes A549 cells to radiation. (A) Expression levels of miR-21 in A549 cells transfected with control miR or antisense (anti)-miR-21. (B) Glucose consumption and (C) lactate production were measured in cells transfected with control miR or anti-miR-21. ${ }^{*} \mathrm{P}<0.05,{ }^{* *} \mathrm{P}<0.01$ and ${ }^{* * *} \mathrm{P}<0.001$ versus the control. (D) A549 parental cells were transiently transfected with control miRNA or anti-miR-21 for $48 \mathrm{~h}$, followed by irradiation $(0,0.5,1,2,4$ and $6 \mathrm{~Gy}$; dose rate, $2 \mathrm{~Gy} / \mathrm{min})$, and a cell survival assay was performed. "P $<0.05$ versus anti-miR-21. Data are presented as the mean \pm standard error.

and a cell survival assay was performed. The survival fraction of the anti-miRNA-21-transfected cells was significantly decreased, as compared with the control group (Fig. 4D). These results indicate a correlation between miR-21 expression and the radiosensitivity of A549 cells to $\gamma$-radiation.
Inhibition of miR-21 resensitizes lung cancer cells to radiation through HIFla regulation. To further investigate whether miR-21 is capable of modulating the sensitivity of radioresistant lung cancer cells, the A549 radioresistant cells were transfected with anti-miR-21 or a negative control (Fig. 5A). 
A

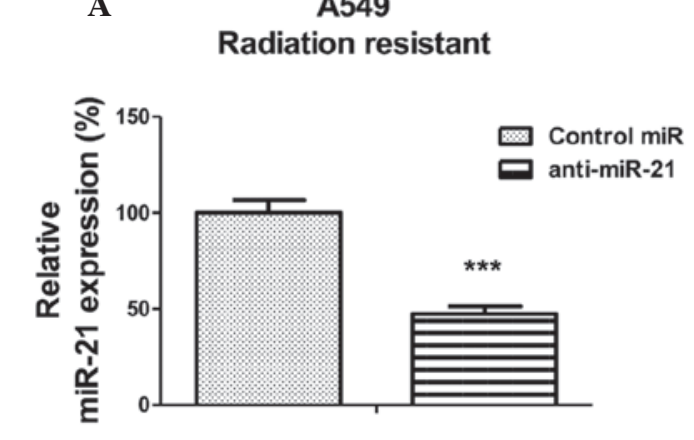

C

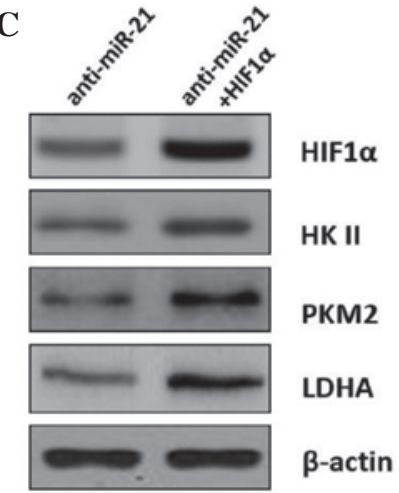

B

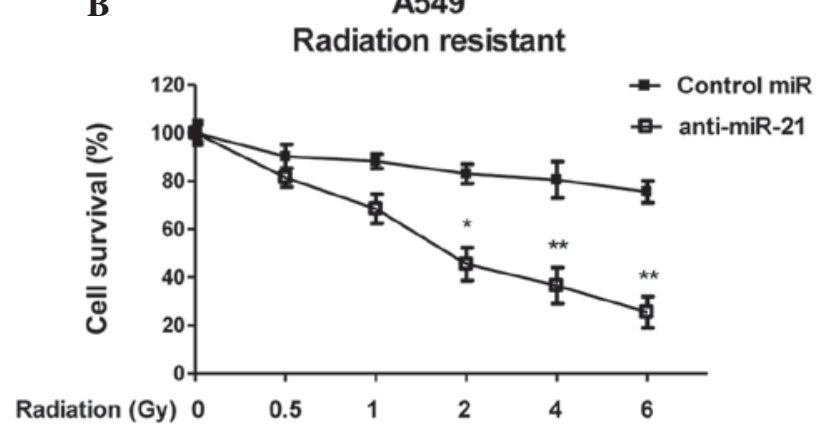

D

Figure 5. Resensitization of radioresistant A549 cells via the inhibition of microRNA (miR)-21-modulated hypoxia-inducible factor (HIF)1 $\alpha$. (A) Expression levels of miR-21 in A549 radioresistant cells transfected with control miR or antisense (anti)-miR-21. ${ }^{* * *} \mathrm{P}<0.001$ versus the control. (B) A549 radioresistant cells were transiently transfected with control miRNA or anti-miR-21 for $48 \mathrm{~h}$, followed by irradiation $(0,0.5,1,2,4$ and $6 \mathrm{~Gy}$; dose rate, $2 \mathrm{~Gy} / \mathrm{min})$, and a cell survival assay was performed. ${ }^{*} \mathrm{P}<0.05$ and ${ }^{* *} \mathrm{P}<0.01$ versus the control. (C) Protein expression of HIF1 $\alpha$, hexokinase (HK)II, pyruvate kinase (PK)M2 and lactate dehydrogenase (LDH)A was rescued following the overexpression of HIF1 $\alpha$ in anti-miR-21-transfected cells, as detected by western blotting. $\beta$-actin was used as a loading control. (D) A549 radioresistant cells were transiently transfected with anti-miR-21 or anti-miR-21 plus HIF1 $\alpha$ for 48 h, followed by irradiation $(0,2,4$ and $6 \mathrm{~Gy}$; dose rate, $2 \mathrm{~Gy} / \mathrm{min})$, and a cell survival assay was performed. Data are presented as the mean \pm standard error. ${ }^{*} \mathrm{P}<0.05$ and ${ }^{* *} \mathrm{P}<0.01$ versus anti-miR-21.

Inhibition of miR-21 significantly increased the susceptibility of the radioresistant cells to irradiation, as compared with the negative control-transfected cells. The half maximal inhibitory concentration $\left(\mathrm{IC}_{50}\right)$ of the anti-miR-21-transfected A549 cells was $2 \mathrm{~Gy}$, which was lower than the $\mathrm{IC}_{50}$ of the negative control-transfected A549 cells, which was 15 Gy (Fig. 5B). To determine the correlation between HIF- $1 \alpha$ and anti-miR-21-induced sensitivity, the anti-miR-21-transfected A549 radioresistant cells were transiently transfected with an overexpression vector containing wild type HIF-1 $\alpha$, in order to rescue its function. With the restoration of HIF1 $\alpha$, the expression levels of the key enzymes of glycolysis were rescued (Fig. 5C). In addition, the sensitivity of HIFl $\alpha$-transfected radioresistant cells to various doses of radiation was determined. As shown in Fig. 5D HIF1 $\alpha$-transfected A549 radioresistant cells regained resistance to radiation. These results suggest that inhibition of miR-21 modulates the sensitivity of A549 radioresistant cells to irradiation via the downregulation of HIF1 $\alpha$.

\section{Discussion}

The present study demonstrated the importance of miR-21-mediated enhanced aerobic glycolysis on acquired radioresistance in non-small cell lung cancer cells. Cancer cells use anaerobic glycolysis to assist in their growth, and a shift toward anaerobic glycolysis occurs with active suppression of oxidative phosphorylation, which results from aberrant regulation of glycolytic enzymes. The present study demonstrated that glycolysis was upregulated in radioresistant cancer cells, suggesting that specific inhibition of glycolysis may contribute to overcoming radioresistance. It has previously been reported that ionizing radiation and various chemotherapeutic drugs induce oxidative stress in targeted cells, leading to genomic instability and lipid peroxidation (24). In addition, excessive lactate production in cancer cells via glycolysis has been reported to act as an antioxidant (25). Therefore, an accumulation of lactate in the tumor microenvironment may jeopardize the sensitivity of cancer cells to radiation and cause chemoresistance.

Upregulation of miR-21 has been detected in numerous types of human cancer. It has previously been reported that miR-21 expression is higher in lung cancer serum samples compared with normal tissues, and high serum miR-21 levels are significantly correlated with lung tumors (19). Furthermore, miR-21 is involved in chemoresistance and radioresistance. A previous study demonstrated that miR-21 was upregulated in human liver cancer following exposure to radiation (26). In human ovarian cancer cells, miR-21 has been show to modulate paclitaxel sensitivity (16). The present study detected miR-21 expression in radiation-resistant and 
radiation-sensitive non-small cell lung cancer cells following exposure to $\gamma$-irradiation. The results demonstrated that miR-21 was upregulated in radioresistant non-small cell lung cancer cells, and inhibition of miR-21 led to resensitization of the radioresistant cells to irradiation, which was consistent with a previous study (19). These data support the hypothesis that miR-21 is not only associated with oncogenesis, but can act as a radioresistant miRNA, which may be considered a therapeutic target for the development of anticancer drugs.

The putative target of miR-21 was screened and HIF1 $\alpha$ was demonstrated to be a potential target. HIF1 $\alpha$ is a multifunctional transcription factor that has been shown to regulate glucose metabolism in a growth factor-dependent manner (23). Previous studies have demonstrated that HIF1 $\alpha$ may contribute to the development of radioresistance (27). As HIF1 $\alpha$ is an upstream signaling molecule of glycolysis, upregulation of HIF1 $\alpha$ promotes glycolysis (23). The results of the present study revealed that HIF $1 \alpha$ was upregulated by miR-21, resulting in the promotion of the key enzymes of glycolysis. Inhibition of HIF1 $\alpha$ by siRNA suppressed glycolysis and resensitized the cancer cells to radiation, thus suggesting that miR-21-induced radioresistance was mediated through the upregulation of HIF1 $\alpha$. However, the detailed mechanisms underlying the miR-21-mediated regulation of HIF $1 \alpha$ remain unclear. In our next project, we aim to investigate this phenotype, and to determine by which pathway miR-21 regulates HIF1 $\alpha$.

In conclusion, the present study detected a strong correlation between the upregulation of miR-21 and radioresistance in non-small cell lung cancer cells. In addition, overexpression of miR-21 activated HIF1 $\alpha$, which stimulated the expression of glycolytic enzymes. Notably, inhibition of miR-21 resensitized radioresistant cancer cells, via the suppression of HIF1 $\alpha$-mediated glycolysis. The present study may provide evidence regarding the targeting of miRNAs for the resensitization of radiation-resistant cancer cells.

\section{Acknowledgements}

The authors of the present study would like to thank the staff and faculty working in the Department of Radiation Oncology, Shandong Cancer Hospital and Institute (Jinan, China), and Dr. Hongjiang Yan for editorial assistance. The present study was supported by a grant from the Science and Technology Department of Shandong Province of China (grant no. 2012YD18087).

\section{References}

1. Ha M and Kim VN: Regulation of microRNA biogenesis. Nat Rev Mol Cell Biol 15: 509-524, 2014.

2. Brodersen P and Voinnet O: Revisiting the principles of microRNA target recognition and mode of action. Nat Rev Mol Cell Biol 10: 141-148, 2009.

3. Ameres SL and Zamore PD: Diversifying microRNA sequence and function. Nat Rev Mol Cell Biol 14: 475-488, 2013.

4. Jansson MD and Lund AH: MicroRNA and cancer. Mol Oncol 6: 590-610, 2012.

5. Sicard F, Gayral M, Lulka H, Buscail L and Cordelier P: Targeting miR-21 for the therapy of pancreatic cancer. Mol Ther 21: 986-994, 2013.
6. Iorio MV, Ferracin M, Liu CG, Veronese A, Spizzo R, Sabbioni S, Magri E, Pedriali M, Fabbri M, Campiglio M, et al: MicroRNA gene expression deregulation in human breast cancer. Cancer Res 65: 7065-7070, 2005.

7. Ma Y, Xia H, Liu Y and Li M: Silencing miR-21 sensitizes non-small cell lung cancer A549 cells to ionizing radiation through inhibition of PI3K/Akt. Biomed Res Int 2014: 617868, 2014.

8. Pineau P, Volinia S, McJunkin K, Marchio A, Battiston C, Terris B, Mazzaferro V, Lowe SW, Croce CM and Dejean A: miR-221 overexpression contributes to liver tumorigenesis. Proc Natl Acad Sci USA 107: 264-269, 2010.

9. Chan SH, Wu CW, Li AF, Chi CW and Lin WC: miR-21 microRNA expression in human gastric carcinomas and its clinical association. Anticancer Res 28: 907-911, 2008.

10. Iorio MV, Visone R, Di Leva G, Donati V, Petrocca F, Casalini P, Taccioli C, Volinia S, Liu CG, Alder H, et al: MicroRNA signatures in human ovarian cancer. Cancer Res 67: 8699-8707, 2007

11. Yao T and Lin Z: MiR-21 is involved in cervical squamous cell tumorigenesis and regulates CCL20. Biochim Biophys Acta 1822: 248-260, 2012.

12. Kanaan Z, Rai SN, Eichenberger MR, Roberts H, Keskey B, Pan J and Galandiuk S: Plasma miR-21: A potential diagnostic marker of colorectal cancer. Ann Surg 256: 544-551, 2012.

13. Chan JA, Krichevsky AM and Kosik KS: MicroRNA-21 is an antiapoptotic factor in human glioblastoma cells. Cancer Res 65: 6029-6033, 2005.

14. Fu C, Dong W, Wang Z, Li H, Qin Q and Li B: The expression of miR-21 and miR-375 predict prognosis of esophageal cancer. Biochem Biophys Res Commun 446: 1197-1203, 2014.

15. Folini M, Gandellini P,Longoni N,Profumo V,Callari M,Pennati M, Colecchia M, Supino R, Veneroni S, Salvioni R, et al: miR-21: An oncomir on strike in prostate cancer. Mol Cancer 9: 12, 2010.

16. Xie Z, Cao L and Zhang J: miR-21 modulates paclitaxel sensitivity and hypoxia-inducible factor-1 $\alpha$ expression in human ovarian cancer cells. Oncol Lett 6: 795-800, 2013.

17. Fang J, Zhou SH, Fan J and Yan SX: Roles of glucose transporter-1 and the phosphatidylinositol 3-kinase/protein kinase B pathway in cancer radioresistance (review). Mol Med Rep 11: $1573-1581,2015$.

18. Shimura T, Noma N, Sano Y, Ochiai Y, Oikawa T, Fukumoto M and Kunugita N: AKT-mediated enhanced aerobic glycolysis causes acquired radioresistance by human tumor cells. Radiother Oncol 112: 302-307, 2014

19. Wang XC, Wang W, Zhang ZB, Zhao J, Tan XG and Luo JC: Overexpression of miRNA-21 promotes radiation-resistance of non-small cell lung cancer. Radiat Oncol 8: 146, 2013.

20. Vander Heiden MG, Cantley LC and Thompson CB: Understanding the Warburg effect: The metabolic requirements of cell proliferation. Science 324: 1029-1033, 2009.

21. Ganapathy-Kanniappan S and Geschwind JF: Tumor glycolysis as a target for cancer therapy: Progress and prospects. Mol Cancer 12: 152, 2013.

22. Young A, Berry R, Holloway AF, Blackburn NB, Dickinson JL, Skala M, Phillips JL and Brettingham-Moore KH: RNA-seq profiling of a radiation-resistant and radiation sensitive prostate cancer cell line highlights opposing regulation of DNA repair and targets for radiosensitization. BMC Cancer 14: 808, 2014.

23. Lu H, Forbes RA and Verma A: Hypoxia-inducible factor 1 activation by aerobic glycolysis implicates the Warburg effect in carcinogenesis. J Biol Chem 277: 23111-23115, 2002.

24. Azzam EI, Jay-Gerin JP and Pain D: Ionizing radiation-induced metabolic oxidative stress and prolonged cell injury. Cancer Lett 327: 48-60, 2012.

25. Le A, Cooper CR, Gouw AM, Dinavahi R, Maitra A, Deck LM, Royer RE, Vander Jagt DL, Semenza GL and Dang CV: Inhibition of lactate dehydrogenase A induces oxidative stress and inhibits tumor progression. Proc Natl Acad Sci USA 107: 2037-2042, 2010.

26. Liang G, Li G, Wang Y, Lei W and Xiao Z: Aberrant miRNA expression response to UV irradiation in human liver cancer cells. Mol Med Rep 9: 904-910, 2014.

27. Harada H, Inoue M, Itasaka S, Hirota K, Morinibu A, Shinomiya K, Zeng L, Ou G,Zhu Y, Yoshimura M et al: Cancer cells that survive radiation therapy acquire HIF-1 activity and translocate towards tumour blood vessels. Nat Commun 3: 783, 2012. 